\title{
Mise à jour du Système canadien de surveillance des maladies à déclaration obligatoire et de son site Web interactif
}

\author{
S Totten ${ }^{1 *}$, A Medaglia ${ }^{1}$, S McDermott ${ }^{1}$
}

\section{Résumé}

Le Système canadien de surveillance des maladies à déclaration obligatoire fournit des données sur les maladies considérées comme prioritaires dans une optique de surveillance et de contrôle en matière de santé publique. Les nombreuses améliorations qui ont été introduites dans son site Web interactif «Maladies à déclaration obligatoire en direct» concordent avec l'engagement du gouvernement du Canada en faveur des données ouvertes. Cet article fait le point sur les changements intervenus dans les définitions de cas depuis leur dernière publication en 2009, et présente les mises à jour dont le site Web interactif a fait l'objet depuis 2013.

Des changements ont été opérés dans les définitions de cas de cinq maladies. Pour I'hépatite $C$, la nouvelle définition de cas établit désormais une distinction entre infection aiguë et chronique. Pour la cyclosporiase, la définition de cas probable exige l'existence d'un lien épidémiologique, tout en précisant que ce lien serait vraisemblablement le résultat d'une exposition à une source alimentaire commune. Pour la rage, la définition de cas probable repose désormais sur la détection d'un anticorps neutralisant la rage plutôt que sur des titres d'anticorps spécifiques. Pour la maladie de Lyme, les nouvelles définitions de cas probable et confirmé intègrent désormais cinq méthodes pour cerner les zones réputées à risque pour la maladie de Lyme plutôt que les zones endémiques. Pour la tuberculose, la nouvelle définition de cas intègre désormais, outre la culture, un test d'amplification des acides nucléiques pour établir le diagnostic.

Le site "Maladies à déclaration obligatoire en direct» est un outil interactif grâce auquel les utilisateurs peuvent créer des figures et des tableaux personnalisés. Depuis la refonte majeure du site en 2013, de nombreuses modifications ont été faites pour améliorer son aspect et sa convivialité. Les figures et les tableaux peuvent maintenant être générés sous forme de fichiers Excel ou PDF. Il est également possible d'exporter de grands ensembles de données vers des fichiers Excel pour effectuer une analyse approfondie. Les définitions de cas du Système canadien de surveillance des maladies à déclaration obligatoire seront mises à jour au besoin, et son site Web interactif continuera d'être amélioré et actualisé en réponse aux commentaires des utilisateurs.

Citation proposée : Totten S, Medaglia A, McDermott S. Mise à jour du Système canadien de surveillance des maladies à déclaration obligatoire et de son site Web interactif. Relevé des maladies transmissibles au Canada 2019;45(10):283-8. https://doi.org/10.14745/ccdr.v45i10a02f

Mots-clés : surveillance, maladies à déclaration obligatoire, définition de cas, Canada, mise à jour

\section{Introduction}

La surveillance des évènements sanitaires est une fonction essentielle de la santé publique. Elle épaule le Canada dans ses efforts visant à améliorer la santé publique à l'échelle nationale et dans le reste du monde. Le Système canadien de surveillance des maladies à déclaration obligatoire (SCSMDO) est géré par
Cette oeuvre est mise à la disposition selon les termes de la licence internationale Creative Commons Attribution 4.0

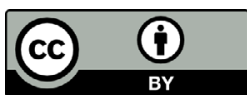

Affiliation

${ }^{1}$ Centre de la lutte contre les maladies transmissibles et les infections, Agence de la santé publique du Canada, Ottawa (Ontario)

\footnotetext{
${ }^{\star}$ Correspondance :

stephanie.totten@canada.ca
}

I'Agence de la santé publique du Canada (ASPC). Ce système national de surveillance exerce un suivi des maladies infectieuses que les gouvernements fédéral, provinciaux et territoriaux ont identifiées conjointement, et fournit des données actuelles et précises pour éclairer les programmes et les politiques en matière 
de santé publique (1). Il fait désormais partie de l'Initiative des données ouvertes du gouvernement du Canada, qui a pour but de procurer à la population canadienne un accès aux données qui sont produites (2), recueillies et utilisées par le gouvernement fédéral. La surveillance est l'une des principales sources de données ouvertes en santé publique.

\section{Contexte}

La collecte nationale de données sur les maladies transmissibles a été entreprise pour la première fois en 1924 par le Bureau fédéral de la statistique (aujourd'hui Statistique Canada). Cette responsabilité a été confiée au Centre de l'hygiène du milieu de Santé Canada en 1988, puis à I'ASPC en 2004, année où l'Agence a vu le jour. Ces données sont transmises et utilisées dans le cadre législatif de la Loi sur la statistique et de la Loi sur le ministère de la Santé.

En vertu des lois provinciales et territoriales, les professionnels de la santé, les hôpitaux et les laboratoires sont tenus de signaler certains cas de maladies aux autorités de santé publique : il s'agit généralement des maladies dites «à déclaration obligatoire». De nombreuses maladies à déclaration obligatoire sont également des maladies à déclaration obligatoire à l'échelle nationale (MDOEN), même si ce n'est pas vrai dans tous les cas. Les autorités provinciales et territoriales de santé publique signalent volontairement au gouvernement fédéral les MDOEN pour faciliter l'agrégation de données et la déclaration à l'échelle nationale.

La liste des MDOEN est tenue à jour en vertu d'un processus de collaboration fédérale-provinciale-territoriale (3-5). On compte à l'heure actuelle 56 MDOEN. Pour la majorité des maladies, les cas sont signalés chaque année au SCSMDO par les autorités provinciales et territoriales, qui transmettent également des renseignements démographiques de base comme l'âge et le sexe. Quelques maladies (VIH/SIDA, tuberculose, virus du Nil occidental, maladie de Creutzfeldt-Jakob, grippe et paralysie flasque aiguë) sont signalées directement aux programmes spécialisés de I'ASPC en raison de la complexité de la maladie ou du système de surveillance.

Les définitions de cas du SCSMDO visent plus à soutenir les activités de santé publique qu'à poser des diagnostics cliniques. Les définitions normalisées de cas de MDOEN ont été élaborées pour la première fois en 1991 en vertu d'un processus fédéral-provincial-territorial, puis révisées en 2000 (6) et en 2009 (7). À la suite de la révision de 2009, il a été décidé que les prochaines mises à jour se feraient au cas par cas.

Un site Web interactif rudimentaire a été lancé pour le SCSMDO en 2001 pour remplacer les rapports annuels jusque-là imprimés. En 2013, le site Maladies à déclaration obligatoire en direct (MDOD) a fait l'objet d'une refonte complète en réponse aux besoins exprimés par les utilisateurs et à la nécessité d'intégrer les nouvelles exigences en matière d'accessibilité. Toutes les données du SCSMDO y ont été intégrées (en remontant jusqu'en 1924, lorsque les données sont disponibles) (1). Depuis 2013, de nouvelles améliorations ont été introduites, y compris une meilleure interactivité et un plus grand choix d'options dans la fonction de diagramme (1). Aujourd'hui, environ 600 utilisateurs individuels consultent le site chaque mois.

\section{Objectifs}

Cet article vise à décrire le processus de mise à jour des définitions de cas de MDOEN, à récapituler les changements introduits dans cinq définitions de cas de MDOEN depuis la révision du SCSMDO en 2009 , et à présenter les dernières améliorations apportées à l'outil interactif d'interrogation en ligne (MDOD).

\section{Comment les définitions de cas de maladies à déclaration obligatoire à l'échelle nationale sont-elles mises à jour}

Les mises à jour des définitions de cas sont coordonnées à travers le Réseau pancanadien de santé publique (RSP). Une révision des définitions de cas peut être entreprise à $n$ 'importe quel moment de l'année dès qu'un besoin de ce type est mis en évidence par les programmes épidémiologiques ou de laboratoire à l'échelle fédérale, provinciale ou territoriale. Entre 2009 et 2013, les définitions ont été mises à jour au travers de collaborations fédérales-provinciales-territoriales informelles, et révisées par le Comité directeur sur les maladies transmissibles et infectieuses (CDMTI) du RSP avant d'être mises en œuvre en vue d'une surveillance à l'échelon national. En 2013, un processus plus structuré a été validé par le Conseil du Réseau pancanadien de santé publique. Ce processus comprend une sollicitation annuelle de manifestation d'intérêt. Lorsque la décision de procéder est prise, un groupe de travail technique est mis sur pied; celui-ci entreprend une révision et présente ses recommandations au CDMTI. Le groupe de travail réunit des experts de laboratoire, des experts en épidémiologie et des experts cliniques, ainsi que d'autres personnes nommées par les membres provinciaux/territoriaux du CDMTI. Une fois validée par le CDMTI, la nouvelle définition de cas est appliquée aux activités de surveillance de routine. En règle générale, la plupart des provinces et des territoires adoptent les définitions de cas nationales pour leur propre usage. II existe néanmoins, pour certaines maladies, de légères différences entre les définitions de cas nationales et celles utilisées dans les activités provinciales/ territoriales de surveillance de la santé publique.

\section{Mise à jour de cinq définitions de cas}

Depuis la dernière publication des définitions de cas du SCSMDO en 2009, cinq révisions ont été apportées aux définitions de cas (8). Trois révisions (hépatite $C$, cyclosporiase, 
rage) ont été opérées avant 2013 et deux révisions (maladie de Lyme et tuberculose) ont été opérées après l'adoption du protocole du Réseau pancanadien de santé publique en 2013.

\section{Hépatite $C$}

La définition de cas nationale adoptée en 2009 pour l'hépatite C (7) ne faisait pas la distinction entre l'infection aiguë et celle chronique. La nouvelle définition comprend une description de l'infection au virus de l'hépatite $C$ aiguë (récemment contractée) en se fondant sur les symptômes, la sérologie et d'autres examens cliniques, ou sur une séroconversion documentée sur une période de douze mois. Tous les autres cas d'hépatite $C$ doivent être signalés dans une catégorie indéterminée qui regroupe les infections chroniques et guéries. Ce changement dans la définition de cas ne devrait pas influer sur le nombre total de cas d'hépatite $C$ signalés chaque année. II servira cependant $d$ 'indicateur du taux d'incidence, permettant ainsi l'analyse des tendances dans les infections récemment contractées. Cette définition de cas a été validée par le CDMTI en 2011.

\section{Cyclosporiase}

La définition de cas adoptée en 2009 pour la cyclosporiase probable (7) a été révisée de manière à mieux concorder avec les autres définitions de cas probable de maladies entériques au Canada et aux États-Unis. Dans le détail, la nouvelle définition précise que les cas probables doivent présenter un lien épidémiologique avec un cas confirmé en laboratoire. Une mention supplémentaire a été ajoutée à la section Commentaires indiquant que la contamination interhumaine directe était improbable et que le lien épidémiologique serait vraisemblablement le résultat d'une exposition à une source alimentaire commune. Ce changement dans la définition de cas ne devrait pas influer sur le nombre total de cas de cyclosporiase signalés à l'échelle nationale, car le SCSMDO ne rapporte que les cas confirmés. Ce changement n'a aucune incidence au niveau des laboratoires, car la définition de cas probable ne se fonde pas sur les critères de laboratoire. Cette définition de cas a été validée par le CDMTI en 2012.

\section{Rage}

La définition de cas adoptée en 2009 pour la rage probable a été modifiée, car les critères de laboratoire prêtaient à confusion. La définition de cas de 2009 se fondait sur des titres d'anticorps spécifiques alors que les seuils d'anticorps n'étaient pas nécessaires au diagnostic de la rage. La référence à un "titre supérieur ou équivalent à cinq" dans la définition de cas probable a été supprimée et remplacée par la phase «détection d'anticorps neutralisant la rage». En outre, la référence à un seuil de détection de $0,5 \mathrm{UI} / \mathrm{ml}$ a été supprimée de la section Laboratoire - Commentaires. Le libellé de la section Laboratoire - Commentaires a également été modifié pour souligner que la sérologie ne doit être utilisée qu'en conjonction avec des tests complémentaires pour le diagnostic de la rage. Ces changements ne devraient pas influer sur le signalement des cas de cette maladie rare. Comme le Réseau des laboratoires de santé publique du Canada avait déjà été consulté et s'était dit d'accord avec tous les changements introduits dans cette définition de cas, celle-ci a été présentée au CDMTI en 2012 dans une optique purement informative.

\section{Maladie de Lyme}

La définition de cas adoptée en 2009 pour la maladie de Lyme (7) contenait des indications très précises pour la détermination d'une zone endémique, ce qui obligeait les provinces et territoires chargés du signalement à exercer une surveillance intensive et à mobiliser des ressources considérables pour ce faire. Il est recommandé de mettre en évidence une preuve d'exposition au risque environnemental lorsqu'on utilise des méthodes sérologiques pour diagnostiquer la maladie de Lyme (9). Ainsi, la nouvelle définition de cas intègre cinq méthodes pour cerner les zones réputées à risque pour la maladie de Lyme, notamment deux méthodes de surveillance active sur le terrain; la surveillance passive des tiques; les signaux des activités de surveillance des cas humains; et les modèles de prévision validés. Des précisions ont également été apportées sur les échantillons cliniques appropriés aux fins de diagnostic en laboratoire. Ces changements devraient permettre d'améliorer la détection du risque associé à la maladie de Lyme ainsi que le signalement des cas de cette maladie par les autorités provinciales et territoriales de santé publique. Ces changements dans la définition de cas nationale devraient accroitre le nombre de cas probables et confirmés avec les cinq méthodes mises en place pour cerner les zones à risque. Cette définition de cas a été validée par le CDMTI en 2016 puis mise en œuvre sur l'ensemble des provinces et territoires à l'exception de la Saskatchewan.

\section{Tuberculose}

La définition de cas adoptée en 2009 pour la tuberculose (7) comprenait uniquement la méthode de culture pour les cas confirmés en laboratoire. La nouvelle définition intègre un test d'amplification des acides nucléiques, cette méthode étant de plus en plus utilisée en laboratoire pour confirmer l'infection à Mycobacterium tuberculosis. Par ailleurs, les cas auparavant classifiés comme "confirmés en clinique" sont désormais classifiés comme "diagnostiqués en clinique», et les critères relatifs à ces cas ont été mis à jour pour tenir compte de ceux utilisés dans la pratique par les provinces et territoires chargés du signalement. Ces changements ne devraient pas influer sur les tendances nationales dans les données de surveillance de la tuberculose. Cette définition de cas a été validée par le CDMTI au debut de 2019 et sera mise en œuvre en janvier 2020 pour la publication des données annuelles sur la tuberculose pour l'année 2019.

Les changements apportés à ces définitions de cas sont récapitulés dans le tableau 1. 
Tableau 1 : Résumé des changements opérés dans les définitions de cas des maladies à déclaration obligatoire à l'échelle nationale : 2009-début 2019

\begin{tabular}{|c|c|c|c|}
\hline $\begin{array}{l}\text { Maladie à } \\
\text { déclaration } \\
\text { obligatoire } \\
\text { à l'échelle } \\
\text { nationale } \\
\text { (année du } \\
\text { changement) }\end{array}$ & Changement & Raison & $\begin{array}{l}\text { Incidence } \\
\text { attendue } \\
\text { sur les } \\
\text { tendances } \\
\text { nationales }\end{array}$ \\
\hline $\begin{array}{l}\text { Hépatite C } \\
\text { (2011) }\end{array}$ & $\begin{array}{l}\text { La définition de cas } \\
\text { établit désormais } \\
\text { une distinction } \\
\text { entre l'infection } \\
\text { aiguë et chronique. }\end{array}$ & $\begin{array}{l}\text { La définition } \\
\text { précédente } \\
\text { manquait de } \\
\text { précisions } \\
\text { qui étaient } \\
\text { disponibles } \\
\text { dans certaines } \\
\text { provinces } \\
\text { et certains } \\
\text { territoires. }\end{array}$ & $\begin{array}{l}\text { Aucune } \\
\text { incidence sur } \\
\text { le nombre } \\
\text { total de cas }\end{array}$ \\
\hline $\begin{array}{l}\text { Cyclosporiase } \\
(2012)\end{array}$ & $\begin{array}{l}\text { La définition de cas } \\
\text { probable précise } \\
\text { désormais que } \\
\text { les cas doivent } \\
\text { présenter un lien } \\
\text { épidémiologique } \\
\text { avec un cas } \\
\text { confirmé en } \\
\text { laboratoire } \\
\text { (vraisemblablement } \\
\text { par l'intermédiaire } \\
\text { d'une source } \\
\text { alimentaire } \\
\text { commune). }\end{array}$ & $\begin{array}{l}\text { Mieux } \\
\text { concorder } \\
\text { avec les autres } \\
\text { définitions de } \\
\text { cas probable } \\
\text { de maladies } \\
\text { entériques au } \\
\text { Canada et aux } \\
\text { États-Unis. }\end{array}$ & $\begin{array}{l}\text { Aucune } \\
\text { incidence }\end{array}$ \\
\hline $\begin{array}{l}\text { Rage humaine } \\
(2012)\end{array}$ & $\begin{array}{l}\text { La définition de } \\
\text { cas probable } \\
\text { repose désormais } \\
\text { sur la «détection } \\
\text { d'un anticorps } \\
\text { neutralisant la } \\
\text { rage » plutôt } \\
\text { que sur des } \\
\text { titres d'anticorps } \\
\text { spécifiques. }\end{array}$ & $\begin{array}{l}\text { Les titres } \\
\text { d'anticorps } \\
\text { spécifiques } \\
\text { ne sont pas } \\
\text { nécessaires } \\
\text { pour le } \\
\text { diagnostic de } \\
\text { la rage. }\end{array}$ & $\begin{array}{l}\text { Aucune } \\
\text { incidence }\end{array}$ \\
\hline $\begin{array}{l}\text { Maladie de } \\
\text { Lyme (2016) }\end{array}$ & $\begin{array}{l}\text { La nouvelle } \\
\text { définition de cas } \\
\text { intègre désormais } \\
\text { cinq méthodes } \\
\text { pour cerner les } \\
\text { zones réputées } \\
\text { à risque pour la } \\
\text { maladie de Lyme, } \\
\text { plutôt que d'exiger } \\
\text { une preuve étayant } \\
\text { l'existence d'une } \\
\text { zone endémique. }\end{array}$ & $\begin{array}{l}\text { La } \\
\text { détermination } \\
\text { d'une zone } \\
\text { endémique } \\
\text { nécessite une } \\
\text { surveillance } \\
\text { intensive qui } \\
\text { mobilise des } \\
\text { ressources } \\
\text { considérables. }\end{array}$ & $\begin{array}{l}\text { Augmentation } \\
\text { du nombre de } \\
\text { cas confirmés } \\
\text { et probables }\end{array}$ \\
\hline $\begin{array}{l}\text { Tuberculose } \\
\text { (2019; en } \\
\text { vigueur en } \\
\text { janvier 2020) }\end{array}$ & $\begin{array}{l}\text { La définition de cas } \\
\text { confirmé intègre } \\
\text { désormais un test } \\
\text { d'amplification des } \\
\text { acides nucléiques } \\
\text { et la définition de } \\
\text { cas clinique a été } \\
\text { affinée. }\end{array}$ & $\begin{array}{l}\text { L'ajout du } \\
\text { TAAN et } \\
\text { l'affinement } \\
\text { de la } \\
\text { définition } \\
\text { s'inspirent } \\
\text { des meilleures } \\
\text { pratiques } \\
\text { actuelles. }\end{array}$ & $\begin{array}{l}\text { Aucune } \\
\text { incidence }\end{array}$ \\
\hline
\end{tabular}

Abréviation : TAAN, test d'amplification des acides nucléiques

\section{Mises à jour apportées à Maladies à déclaration obligatoire en direct}

Depuis la refonte du site interactif MDOD en 2013, les mises à jour annuelles ont permis d'améliorer son aspect et sa convivialité. En 2017, une fonction a été ajoutée permettant aux utilisateurs de télécharger sous forme de fichier PDF ou de tableau Microsoft Excel les données en ligne issues de leur recherche. En 2018, une nouvelle fonction de diagramme a été introduite avec de nombreuses options pour la sortie des données. Par exemple, pour obtenir un simple diagramme des tendances nationales dans les infections à Campylobacter et à Salmonella entre 1991 et 1996, il suffit à l'utilisateur d'indiquer ces deux maladies et de préciser la période visée, et un diagramme illustrant les tendances au cours du temps sera automatiquement généré (appendice 1a). Des diagrammes personnalisés peuvent également être créés selon les caractéristiques fournies (par exemple, définition des axes et groupement des variables) et des filtres peuvent être appliqués suivant les besoins (par exemple, par groupe d'âge, sexe et année). L'un des avantages de la personnalisation est la capacité à visualiser les données relatives à plusieurs maladies, groupes d'âge et catégories de sexe, en fonction des besoins de l'utilisateur (appendice 1b). Ces diagrammes simples et personnalisés peuvent ensuite être exportés en format PDF ou Excel.

Il est désormais possible d'extraire un grand ensemble de données agrégées, y compris une partie ou l'intégralité des données disponibles sur les MDOEN, et ce, pour toutes les années disponibles depuis 1924. Cette fonction permet de créer un classeur Excel intégrant l'ensemble des données, dans lequel les limites des données et les descriptions des maladies figurent sous l'onglet «Notes» et le tableau des données sous l'onglet «Données». Les données ainsi extraites peuvent être ventilées par âge et par sexe depuis l'année 1991 jusqu'à maintenant. Les mises à jour prévues pour la fin 2019 comprennent la possibilité d'exporter des fichiers de valeurs séparées par des virgules à partir de la fonction d'extraction des données volumineuses, ce qui facilitera l'importation dans les progiciels d'analyse statistique, ainsi que l'amélioration de la visualisation des variations dans la communication des données, ce qui permettra à l'utilisateur de déterminer, d'un simple coup d'œil, si les données concernant une maladie donnée sont représentatives au niveau national.

\section{Conclusion}

Le SCSMDO et le site MDOD fournissent des données ouvertes sur les 56 maladies à déclaration obligatoire au Canada, et le site Web interactif a été amélioré pour répondre aux besoins des utilisateurs. Les nouvelles définitions de cas et l'optimisation des fonctionnalités du site Web ont permis d'améliorer la capacité des personnes intéressées par ces données à y accéder et à créer 
des tableaux et des figures utiles et adaptés à leurs besoins en matière d'information. Ces données peuvent contribuer à faire mieux connaître les tendances dans les maladies infectieuses et à documenter l'élaboration et l'évaluation des programmes et politiques de santé publique à l'échelle nationale. Cette base de données accessible s'inscrit également dans le cadre de l'engagement du gouvernement du Canada en faveur des données ouvertes et de la science ouverte, dont l'objectif global est de renforcer la capacité nationale à prévenir, à atténuer et à lutter contre les maladies infectieuses au Canada. Le site Web continuera d'être amélioré. Les utilisateurs du SCSMDO et du site MDOD sont encouragés à proposer d'autres améliorations en écrivant à l'adresse phac.nd-mado.aspc@canada.ca.

\section{Déclaration des auteurs}

S. T. - Rédaction de la version préliminaire, révision et édition A. M. - Révision et édition

S. M. - Révision et édition

\section{Conflit d'intérêts}

Aucun.

\section{Remerciements}

Nous tenons à remercier les fournisseurs de données provinciaux et territoriaux du Système canadien de surveillance des maladies à déclaration obligatoire (SCSMDO) pour leur collaboration annuelle dans la transmission des données, ainsi que les secteurs des programmes sur les maladies de la Direction générale de la prévention et du contrôle des maladies infectieuses de l'Agence de la santé publique du Canada pour leur contribution à la révision des définitions de cas.

\section{Financement}

Ces travaux ont été soutenus par l'Agence de la santé publique du Canada.

\section{Références}

1. Agence de la santé publique du Canada. Maladies à déclaration obligatoire en direct. ASPC; Ottawa (ON) : 2018. https://maladies.canada.ca/declaration-obligatoire/

2. Gouvernement du Canada. Plan d'action national du Canada pour un gouvernement ouvert de 2018-2020. https://ouvert. canada.ca/fr/contenu/plan-daction-national-du-canada-po ur-un-gouvernement-ouvert-de-2018-2020

3. Carter A and The National Advisory Committee on Epidemiology Subcommittee. Establishing goals, techniques and priorities for national communicable disease surveillance. Can J Infect Dis. 1991; 2(1):37-40. DOI PubMed

4. Doherty JA; ACE Subcommittee on Communicable Diseases. Establishing priorities for national communicable disease surveillance. Can J Infect Dis 2000 Jan;11(1):21-4. DOI PubMed

5. Doherty JA. Rapport final et recommandations du groupe de travail National sur les maladies à déclaration obligatoire. Relevé des maladies transmissibles au Canada 2006 Oct;32(19):211-25. https://www.canada.ca/content/dam/ phac-aspc/migration/phac-aspc/publicat/ccdr-rmtc/06pdf/ cdr3219.pdf

6. Comité consultative de l'épidémiologie et la Division de la surveillance des maladies. Santé Canada. Définitions de cas des maladies faisant l'objet d'une surveillance nationale. Relevé des maladies transmissibles au Canada. 2000; 26 S3. http://publications.gc.ca/collections/Collection/H12-21-3-263F.pdf

7. Agence de la santé publique du Canada. Définitions nosologiques des maladies transmissibles faisant l'objet d'une surveillance nationale. Relevé des maladies transmissibles au Canada. 2009; 35S2. https://www.canada. $\mathrm{ca} /$ content/dam/phac-aspc/migration/phac-aspc/publicat/ ccdr-rmtc/09pdf/35s2-fra.pdf

8. Agence de la santé publique du Canada. Liste actuelle des maladies à déclaration obligatoire à l'échelle nationale et année ou années au cours desquelles la maladie a été rendue déclarable. ASPC; Ottawa (ON): 2018. https://maladies.canada.ca/declaration-obligatoire/ liste-maladies

9. Canadian Public Health Laboratory Network. The laboratory diagnosis of Lyme borreliosis: guidelines from the Canadian Public Health Laboratory Network. Can J Infect Dis Med Microbiol. 2007;18:145-8. DOI PubMed 
Annexe 1a : Exemple de résultat tiré de Maladies à déclaration obligatoire en direct - campylobactériose et salmonelle, taux par 100000 habitants, de 1991 à 2016

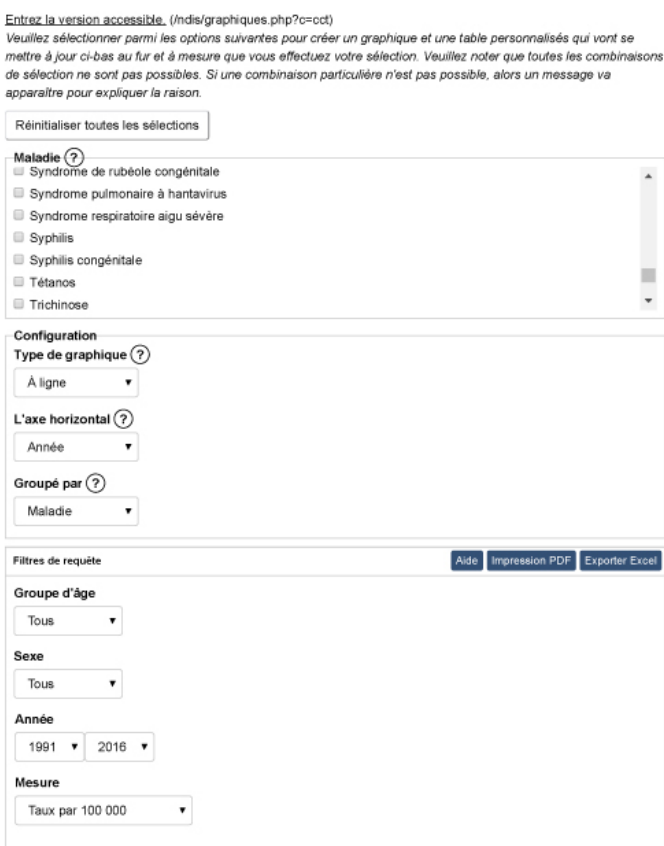

Taux par 100000 des cas signalés de maladies au fil du temps au Canada, groupé par maladie

Tous les âges (incluant les indéterminés), tous les sexes (incluant les

inconnus), 1991-2016

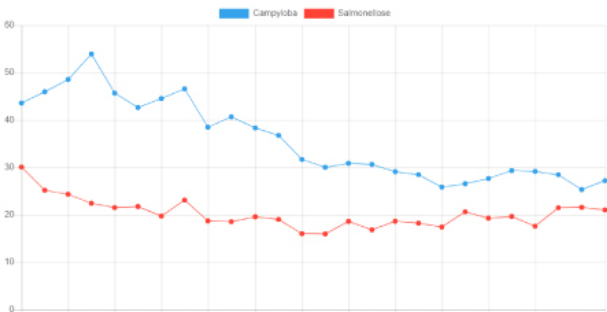

Taux par 100000 des cas signalés de maladies au fill du temps au Canada, groupé par maladie.

tous les àges (incluant les indéterminés), tous les sexes (incluant les inconnus), 1991-2016

\begin{tabular}{|c|c|c|}
\hline Année & Campyloba & Salmonellose \\
\hline 1991 & 43.58 & 30.07 \\
\hline 1992 & 45.96 & 25.17 \\
\hline 1993 & 48,53 & 24,34 \\
\hline 1994 & 53,93 & 22.43 \\
\hline 1995 & 45,68 & 21,52 \\
\hline 1996 & 42,65 & 21,74 \\
\hline 1997 & 44,54 & 19,73 \\
\hline 1996 & 46.58 & 23,11 \\
\hline 1999 & 38,49 & 18,72 \\
\hline 2000 & 40,66 & 18,55 \\
\hline 2001 & 39.32 & 19.58 \\
\hline 2002 & 36,75 & 19,03 \\
\hline 2003 & 31,69 & 16.01 \\
\hline 2004 & 30.02 & 15.96 \\
\hline 2005 & 30,85 & 18,63 \\
\hline 2006 & 30,64 & 16.82 \\
\hline 2007 & 29,09 & 18,66 \\
\hline 2008 & 28,47 & 18.24 \\
\hline 2009 & 25,84 & 17,43 \\
\hline 2010 & 26.55 & 20.63 \\
\hline 2011 & 27.65 & 19.28 \\
\hline 2012 & 29,35 & 19.65 \\
\hline 2013 & 29,16 & 17,6 \\
\hline 2014 & 28,46 & 21,49 \\
\hline 2015 & 25,33 & 21,58 \\
\hline 2016 & 27,21 & 21,03 \\
\hline
\end{tabular}

Annexe $1 \mathrm{~b}$ : Exemple de résultat tiré de Maladies à déclaration obligatoire en direct - chlamydia et gonorrhée par groupe d'âges, taux par 100000 habitants, 2016

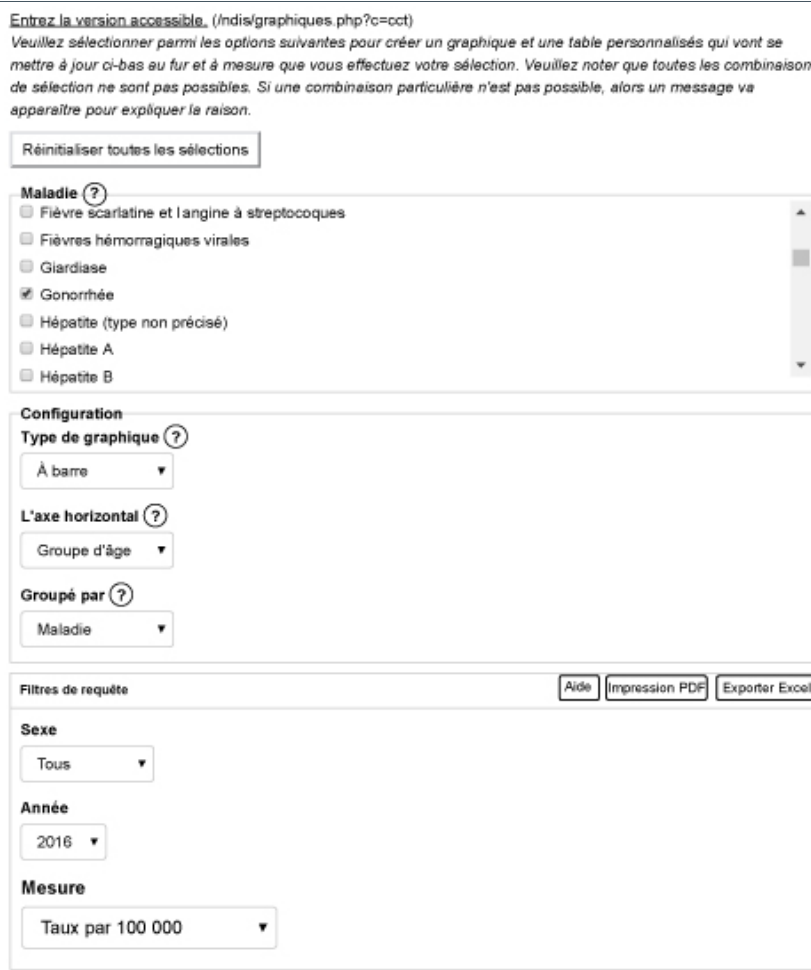

Taux par 100000 des cas signalés de maladies par groupe d'âge au Canada, groupé par maladie

Tous les sexes (incluant les inconnus), 2016

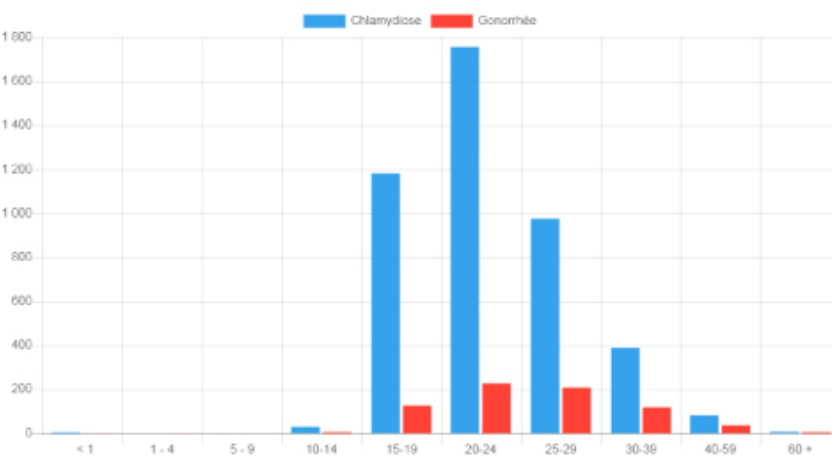

Taux par 100000 des cas signalés de maladies par groupe d'âge au Canada, groupé par maladie, tous les sexes (incluant les inconnus), 2016

\begin{tabular}{|c|c|c|}
\hline Groupe d'äge & Chlamydiose & Gonorrhée \\
\hline$<1$ & 3,88 & 0,52 \\
\hline $1-4$ & 0,13 & 0,39 \\
\hline $5-9$ & 0,45 & 0,15 \\
\hline $10-14$ & 30,48 & 4,67 \\
\hline $15-19$ & 1182,25 & 126,55 \\
\hline $20-24$ & 1756,67 & 228,02 \\
\hline $25-29$ & 975,44 & 208,63 \\
\hline $30-39$ & 388,57 & 119,52 \\
\hline $40-59$ & 81,82 & 36,95 \\
\hline $60+$ & 7,08 & 4,8 \\
\hline
\end{tabular}

